\title{
Las leyes de la didáctica y la realidad escolarizada. ¿Necesidad de cambio?
}

\section{Didactic laws and scholar reality. The change is needed?}

Walfredo González Hernández*

\section{RESUMEN}

Se realiza un análisis de la formación de las ciencias y de la didáctica, así como su categoría fundamental. Se hace un análisis de las leyes de la didáctica y su carácter parcial en la explicación de los fenómenos de la realidad escolarizada, cuestión esta que lleva a definir dos nuevas leyes de esta ciencia.

\section{ABSTRACT}

Paper that analyzes sciences and Didactics development, even its fundamental category. It is analyzed too, Didactics law and its partial explication about the schooled realities phenomena, conducting to define two new laws for a Didactics as a science.

Key words: didactics laws, configurational, complexity

\section{INTRODUCCIÓN}

Las ciencias han recorrido un largo camino desde sus inicios como filosofía y la contemplación del mundo como método fundamental hasta la actualidad, en la cual se reconocen las más disímiles ramas científicas con sus respectivos métodos. Para Núñez (2003) se le puede analizar como el sistema de conocimientos que modifica nuestra visión del mundo real y enriquece nuestro imaginario y nuestra cultura. También se le puede comprender como proceso de investigación que permite obtener nuevos conocimientos, los que a su vez ofrecen posibilidades nuevas de manipulación de los fenómenos; es posible atender a sus impactos prácticos y productivos, caracterizándola como fuerza productiva que propicia la transformación del mundo y es fuente de riqueza. Al mismo tiempo, la ciencia también se presenta como una

\footnotetext{
" Doctorado en Ciencias Pedagógicas de la Universidad de Matanzas, Camilo Cienfuegos, Cuba; Walfredo.glez@umcc.cu,walfredogh@gmail.com
} 
profesión debidamente institucionalizada, portadora de su propia cultura y con funciones sociales bien identificadas. Sin embargo, se reconoce la necesidad de profundizar en este concepto considerándola como una actividad social y cultura al mismo tiempo. Al respecto Kuhn (2004) considera que la ciencia se comporta como una subcultura sostenida por la actividad comunal de grupos practicantes, lo cual hace pensar en la relación dialéctica que se establece entre sus practicantes, el producto resultante y la realidad que pretende describir. Núñez entiende la ciencia

no solo como un sistema de conceptos, proposiciones, teorías, hipótesis, etc., sino también, simultáneamente, como una forma específica de la actividad social dirigida a la producción, distribución y aplicación de los conocimientos acerca de las leyes objetivas de la naturaleza y la sociedad. Aún más, la ciencia se nos presenta como una institución social, como un sistema de organizaciones científicas, cuya estructura y desarrollo se encuentran estrechamente vinculados con la economía, la política, los fenómenos culturales, con las necesidades y las posibilidades de la sociedad dada (2003: 37).

En este artículo se considera necesario agregar que en la ciencia también se abordan las leyes del pensamiento, como en el caso de la psicología, y que se produce conocimiento científico como resultado de esta actividad.

Para Ginoris (2009), el conocimiento científico se caracteriza y reconoce por sistemas teóricos que tienen su origen en métodos propios y rigurosos de la investigación. El conocimiento científico es objetivo, sistémico, universal, exacto y está dirigido a ofrecer explicaciones, predecir y transformar. Es un conocimiento que evoluciona en un proceso dialéctico entre verdades absolutas y verdades relativas. A diferencia del conocimiento filosófico, el científico intenta descubrir las relaciones y causas específicas de los diferentes tipos de hechos, fenómenos y procesos de la naturaleza, la sociedad y el hombre. De aquí se comprenden los diferentes intentos de clasificar u ordenar las ciencias.

Una de las ciencias que más tardíamente se ha separado de la filosofía es la didáctica. Aún existen fuertes polémicas por su carácter de ciencia debido a las diversas consideraciones que sobre 
ella existen en la literatura de diversa índole. Sin embargo, la didáctica, gradualmente, ha ido ganando su espacio en el conjunto de ciencias que existen en la actualidad a partir de la definición de su objeto de estudio.

Varios autores (Addine, 2010; Fuentes y Álvarez, 2003; Ginoris, 2009; Ginoris, Addine, y Turcaz, 2006; Klimber, 1988) reconocen el objeto de estudio de la didáctica como el proceso de formación escolarizada del hombre, lo cual excluye, según Ginoris (2009), procesos que se desarrollan en la familia o en la comunidad. Otro elemento distintivo de las ciencias, y la Didáctica no escapa a ello, es poseer un conjunto de leyes, principios y categorías para conformar su cuerpo teórico.

De los referentes teóricos sobre ciencia analizados hasta el momento se puede inferir que las disciplinas científicas deben ser modificadas en la misma medida en que cambian las explicaciones sobre los fenómenos de la realidad o en que existan fenómenos que no es capaz de explicar. ¿Será este el caso de la Didáctica como ciencia?

Las ciencias naturales y exactas, en los últimos ańos, han presentado un cuadro del mundo totalmente diferente al que se basaba en el orden y la gradualidad. Los cambios abruptos, la imposibilidad de predicción, la pobre clasificación de fenómenos, entre otros, han marcado las pautas en el pensamiento científico actual. Sería interesante analizar si este fenómeno se presenta de manera análoga en el proceso de formación escolarizado, objeto de estudio de la didáctica. Es opinión de este autor que existen elementos que hacen pensar de esta manera, entre los cuales se puede destacar la emergencia de los líderes escolares, los sistemas educativos que forman individuos no acordes con el contexto social en el cual se desarrollan, entre otros ejemplos. Es entonces cuando las leyes de la Didáctica, presentes en la literatura cubana al respecto, merecen un nuevo análisis desde varias aristas, siendo el paradigma complejo una de ellas. 


\section{DESARROLLO}

\section{La Didáctica como ciencia y la concepción compleja de la realidad}

Cuando se analiza el concepto de ley, como parte del cuerpo teórico de una ciencia, existen variadas definiciones. Una de ellas la aporta Afanasiev (1981: 59) cuando plantea que es "un nexo esencial y necesario, general y reiterable entre los fenómenos del mundo material que permite una rigurosa determinación del curso de los acontecimientos”. De esta definición se desprende la relación simbiótica que existe entre las leyes y la realidad.

$\mathrm{Al}$ asumir una postura didáctica referente a las ciencias, Ginoris plantea que:

las leyes que se han logrado identificar en el proceso de enseñanza-aprendizaje escolarizado rigen en todas las características, relaciones y desarrollo del fenómeno al cual pertenecen. Esta cualidad de las leyes científicas posee enorme valor metodológico para comprender, explicar, predecir o planear, conducir y evaluar y hasta investigar, en el caso que nos ocupa, el proceso de enseñanza-aprendizaje. Las leyes que se reconocen aquí no son producto de decisiones humanas, son leyes inherentes del proceso de enseñanza-aprendizaje $y$, por lo tanto, son universales para todo proceso de enseñanza-aprendizaje escolarizado; sin exclusión ni excepciones. Además, son asumidas como leyes objetivas que, al ser conocidas y consideradas, permiten la conducción científica de la enseñanza y el aprendizaje en las instituciones docentes (2009: 12).

De la cita anterior se fundamenta la definición de una de las leyes de la Didáctica con la cual concuerdan todos los autores revisados (Addine, 2010; Álvarez, 1995; Bastart, Reyes, y González, 2013; Concepción, Porbén, y Saíz, 2012; Edel, 2011; Ginoris, 2009; Gómez, 2012; Müller, 2008): el proceso de enseńanzaaprendizaje está determinado por el contexto sociohistórico. Para Álvarez (1995), en esta ley se expresa la relación entre el encargo social y el objetivo de la educación. Sin embargo, se pueden encontrar otras consideraciones. Maldonado considera que: 
En efecto, aunque suene trivial, la educación es un fenómeno esencialmente dinámico que sucede, cada vez más, en el mundo contemporáneo, en entornos cambiantes y en tiempos caracterizados por turbulencias. Por lo tanto, el aula de clase, seminario, taller o laboratorio pueden y deben ser vistos, adecuadamente, como sistemas abiertos sensibles a los procesos, estructuras y dinámicas del entorno. La formación y la educación -en la distinción mencionada- tienen lugar exactamente en estas circunstancias, responden a ellas y esas mismas fluctuaciones se plasman en la educación, directa o indirectamente (2014: 11).

Cuando se trata de la categoría más importante de la Didáctica: el proceso de enseñanza-aprendizaje (Ginoris, 2009), es posible afirmar que este proceso no es lineal y que en él pueden intervenir múltiples factores. Siendo consecuente con las ideas del párrafo anterior, el aprendizaje de los actores que intervienen en el proceso puede expresarse con un alto grado de indeterminación, elemento característico de los fenómenos complejos (Almeida, 2008; Arecchi, 2010; Boon, Casti, y Taylor, 2011; Chae, Seo, y Lee, 2013; Jörg, 2010; Marion, 2012; Mason, 2008; Mitjáns, 2010; Richardson, 2010; Wang, Tsai, y Tsai, 2014) y que debe ser de especial atención en la Didáctica, tal y como se concibe en Cuba. El ser consecuente con la psicología de orientación dialéctico materialista (Díaz y González, 2005; González, 2000, 2010) lleva a reconocer la complejidad del aprendizaje como parte esencial en la formación de la subjetividad humana, proceso siempre en movimiento, no lineal, para la apropiación de la cultura humana (Entel, 2000; Larsson y Dahlin, 2012).

Si en se asume una de las consecuencias de la primera ley mencionada de la Didáctica: la educación responde al encargo social, se infiere entonces que su producto: la formación del ser humano, debe responder a la sociedad en la cual vive. Sin embargo, a partir de esta inferencia, no es posible explicar cómo surgen los líderes sociales que han conducido revoluciones sociales, científicas o de otra índole como Einstein, da Vinci, Gandhi, Roosevelt o Fidel. De manera general, en el proceso de enseñanza-aprendizaje, no siempre se cumple el encargo social, pero esta afirmación de la realidad contradice la primera ley. 
La opinión del autor es que la primera ley es la aspiración del conjunto de autores ya mencionados, pero no es aplicable de manera inexorable al proceso. En este análisis la existencia de las figuras relevantes, tomadas como ejemplos en el párrafo anterior, contradice el planteamiento de Ginoris (2009) que son leyes inherentes del proceso de enseńanza-aprendizaje y, por lo tanto, son universales para todo proceso de enseñanza-aprendizaje escolarizado; sin exclusión ni excepciones. Es en estos casos, según Kunh (1984), que el paradigma predominante no estudia estas anomalías en su función predictiva de la realidad y es necesario que sea modificado de tal manera que logre explicarla de manera conclusiva.

La realidad ya es rebelde al conocimiento, como fue concebido por Descartes, no se deja subdividir ni clasificar, es holística y trandisciplinar, cuestión esta difícil de comprender sin un nuevo paradigma que lo sustente: la complejidad. En la filosofía de las ciencias se está pasando de una filosofía del continuo, de las aproximaciones graduales en el tiempo, de las leyes deterministas a una filosofía del abrupto, de la relación entre la causalidad y la casualidad (con las consecuencias que esto trae). En esta concepción del mundo y sus relaciones, en las cuales las causas pueden ser factores y los factores convertirse en causas, de relaciones no lineales en el tiempo y en el espacio, de crisis como procesos donde hay un surgimiento de nuevos procesos y su relación con los procesos graduales de cambios cualitativos en cuantitativos, la Didáctica, como sistema teórico, debe ser reformulada.

Todo ello puede expresar la necesidad de introducir el paradigma de la complejidad en el contexto educativo, como aprecian diversos autores (Jörg, 2010; Larsson y Dahlin, 2012; López, 2012; Marion, 2012; Mason, 2008; Ricca, 2012; Richardson, 2010; Trombly, 2014) para estudiar fenómenos que no han sido tratados actualmente. Ello no quiere decir que el proceso docente educativo sea un proceso caótico, como se concibe en la ciencia normal (Kuhn, 1984), sino un proceso en el cual se sucede el orden y el desorden como expresiones del desarrollo en espiral y para lo cual el sistema educativo debe estar preparado. Este nuevo paradigma no excluye los sistemas teóricos conocidos, sino que los incorpora a su visión del mundo y sus relaciones. Se asume 
que la didáctica no es simple, ordenada, estática (acabada) y lineal, tal como lo muestran la mayoría de los textos sobre didáctica ya declarados, en consonancia con la mecánica newtoniana, sino contradictoria, holística, integradora, no lineal y llena de rupturas en su praxis y teoría.

Para explicar lo complejo de los procesos de enseńanza-aprendizaje es necesario comprender las diferentes tipologías de problemas que, dentro del paradigma de la complejidad, coexisten y deben ser explicitados para su posterior análisis en la Didáctica. Para Reynoso:

se acuña el término de complejidad organizada para referirse a aquellos fenómenos o problemas donde interviene un número amplio de factores o variables interrelacionados que conforman un todo orgánico. En otros términos, abordar el problema de la organización implica dar cuenta de la génesis y emergencia de totalidades complejas, que no resultan comprensibles por medio de enfoques reduccionistas-analíticos. Mientras que los problemas de simplicidad pueden ser abordados por modelos mecánicos, y los problemas de complejidad desorganizada pueden ser estudiados por medio de modelos estadísticos; los problemas de complejidad organizada son abordables por modelos sistémicos (2006: 24).

De esta tipología se asume la didáctica como complejidad organizada, al reconocer la multiplicidad de factores y variables que la constituyen, el carácter sistémico de sus elementos articulados alrededor de su categoría principal: proceso de enseñanza-aprendizaje escolarizado.

En la concepción compleja de la realidad aparece el concepto de improbabilidad, el cual es defendido en las obras relacionadas con el desarrollo biológico. Al respecto se plantea que "el rasgo más fuerte de los sistemas complejos se revela como el de su impredecibilidad. Este rasgo altera magníficamente el estatuto mismo de la ciencia y de la racionalidad humana en general." (Maldonado, 2003: 15). La impredecibilidad en los procesos de enseñanza-aprendizaje se fundamenta al tomar en cuenta la multiplicidad de factores que intervienen en ella, a los cuales Ginoris hace referencia cuando habla de la actuación del docente de la siguiente manera: "pero aunque no son realmente parte de la 
teoría científica por ser elementos diversos, personales, no universales, subjetivos y muchas veces dados por experiencias únicas, se les considera complementos de la teoría didáctica" (2009: 13), cuestión esta que no se comparte en este artículo al alejar premeditadamente parte de la realidad en el proceso de abstracción que se hace de la misma.

Uno de los componentes de la impredecibilidad lo constituyen los actores que intervienen en el proceso didáctico desde las condiciones históricas concretas en que se desarrollan, su concepción sobre el proceso, su preparación para enfrentar el aprendizaje de sus estudiantes, entre otras cuestiones, que necesita de un estudio holístico y configuracional (Fuentes y Álvarez, 2003) en su concreción práctica. Asumir, hasta el momento, el enfoque holístico y configuracional propuesto por estos autores en la teoría y práctica didáctica conlleva a la solución de la complejidad funcional inherente a la impredecibilidad del proceso de enseñanza-aprendizaje.

En este sentido, las planificaciones centradas en el tiempo y los objetivos eminentemente secuenciales (Maldonado, 2014), las estructuras rígidas y esquemáticas que no permiten los procesos altamente subjetivos e individualizados que trascurren en un aprendizaje real, que pretenda ser creativo (González, 2013a, 2013b) y posibilite una formación integral que contemple la investigación como parte esencial en la obtención del conocimiento (Almeida, 2008; Arecchi, 2010; Maldonado, 2014; Mason, 2008; Mitjáns Martínez, 2010; Richardson, 2010) deberán ser eliminadas de la práctica escolar. Es por ello que, reconocer el error como base del pensamiento científico y como parte indisoluble del proceso de aprendizaje, constituye una prioridad para el cual, increíblemente, no se planifica tiempo en la escuela hoy (González y Kanhime, 2009; Kanhime, 2012; Labarrere, 1987) ni en la teoría que se construye al respecto.

Se puede enseñar a pensar, pero es un proceso altamente individualizado a la cual los clásicos componentes del proceso de enseñanza-aprendizaje (Addine, 2010) no dan respuesta cabal. En los componentes descritos en la literatura pedagógica cubana al respecto y en la práctica escolar es difícil de encontrar la aplicación consecuente del concepto de vivencia a pesar de ser un re- 
sultado del enfoque histórico cultural. De ahí que los autores que asumen la determinación del proceso de enseñanza-aprendizaje al contexto sociohistórico como ley, no son lo suficientemente claros al abordar la relación compleja entre el contexto sociohistórico, la propia de la psiquis del estudiante, que se expresa en la subjetividad (Díaz Gómez y González Rey, 2005; González Rey, 2000, 2010) y sus relaciones con otros estudiantes y el sistema educativo. Esta relación no es hoy, en la Didáctica, un problema suficientemente resuelto desde una postura teórica. Se coincide con varios autores (Díaz Gómez y González Rey, 2005; González Rey, 2000, 2010) en que es un proceso totalmente alejado del equilibrio, al constituir un reflejo subjetivo del contexto sociohistórico totalmente diferente para cada estudiante. No reconocer las variaciones que esta relación conlleva a los sistemas educativos a no reconocer la historicidad del proceso educativo ni la historia de la pedagogía en sí misma.

\section{La primera ley de la Didáctica como sistema complejo: la relación configuracional entre la sociedad y la educación}

Como asumen Addine (2010); Álvarez (1995); Gibert (2012); Ginoris (2009); Ginoris et al. (2006), entre muchos otros, el proceso de enseñanza-aprendizaje interactúa con otros actores ajenos al proceso, que intervienen en el mismo de manera diversa y, en ocasiones contradictorias, con el proceso escolar. Ello lleva a pensar que corresponde más a la realidad un equilibrio entre estos sistemas dinámicos: la sociedad y la escuela como parte de ella, con características de no linealidad, dinamismo y abertura a otros sistemas para lograr el desarrollo de la personalidad del individuo.

Es importante detenerse en la relación escuela-sociedad, ya que en la gran mayoría de los libros de didáctica de autores cubanos se asume la interacción en una sociedad. Esta relación, contenida en la primera ley definida en la ciencia considerada como normal por Khun (1984), está en contradicción con la multiculturalidad presente en la sociedad actual, con gran influencia de las redes informáticas que permiten la interacción social en modelos de comunicación y desarrollo más holísticos e integradores como 
el modelo de Engeström (2007, 2008), basado en el enfoque histórico cultural, que amplía y enriquece. Desde esta perspectiva, la relación escuela-sociedad ya no es tan lineal, coherente y exenta de contradicciones como se expresa en los libros y artículos de Didáctica citados en este artículo, ya que la interacción de los participantes en el proceso de enseńanza-aprendizaje escolarizado con la sociedad puede darse en dos sociedades contradictorias entre sí. De esta manera, en un mundo cada vez más interconectado y globalizado, entendido de la manera más general posible y no como globalización neoliberal, las interacciones entre la escuela y sociedad deben ser flexibles, tolerantes a las emergencias y, sobre todo, abiertas a la interacción con otros sistemas tan dinámicos y no lineales como ellos, como puede ser el contexto internacional.

De la propia definición anteriormente planteada se deriva que asumir el paradigma de la complejidad permite a la Didáctica ser una ciencia que no esté separada de la realidad que le dio origen y considerar en ella los elementos subjetivos, cambiantes e inesperados que surgen en el accionar didáctico. Es en este accionar donde se integran de manera holística y configuracional (Fuentes y Álvarez, 2003) los componentes personales y no personales del proceso que se expresan en la amplia teoría al respecto. Las diversas configuraciones que se dan en la práctica dependen de muchos factores objetivos y subjetivos, los cuales pueden llegar a constituir un sistema no lineal.

Para continuar las ideas fundamentales considera el autor imprescindible el análisis del paradigma configuracional de la Didáctica. Fuentes define este paradigma como:

aquellos elementos dinámicos del objeto (sistema de procesos), que tienen una delimitación subjetiva, aunque con un sustento objetivo, se relacionan unos con otros transformándose o interactuando de manera tal, que se integran como un todo en configuraciones de mayor orden, pero no como elementos diferentes que se incorporan, sino como expresiones del todo (1999: 37).

En esta definición no se aprecia cuáles son las condiciones necesarias para la aparición de la configuración, así como la manera en que se integran los elementos constituyentes. Además, este 
autor lo delimita en el plano subjetivo, obviando de esta manera que también es configuracional el actuar de los componentes personales y no personales involucrados en el accionar didáctico.

Para Autores, la configuración se asume como "la organización particular que adoptan sus componentes en un sujeto" (2004: 23) para realizar el análisis de la categoría personalidad; sin embargo, esta definición adolece de ambigüedades. González define configuración en el orden psicológico "como sistema de formaciones diferentes, donde un mismo elemento psicológico parcial puede aparecer de forma simultánea en unas u otras de dichas formaciones, incluso con un sentido psicológico diferente”. (1993: 33). De esta definición se infieren elementos importantes de la organización de la configuración. Uno de ellos es el sistema de formaciones y se infiere que depende de determinadas situaciones cuando continúa expresando "la naturaleza configuracional de la personalidad radica en la integración de contenidos psicológicos cognitivos y afectivos en una nueva realidad" (ibid.: 34). Esta última afirmación vincula la configuración a la realidad sin especificar cuáles son los aspectos de la realidad que originan su organización.

Sin embargo, Ortiz se refiere a la personalidad como "una configuración de contenidos subjetivos que tienen una real significación personal" (2008: 40), aportando el porqué la configuración adquiere estas características, en este caso la personalidad, en dependencia de la significación que la realidad y que adquiere en su interacción con ella. Los autores analizados anteriormente no explican cómo se estructura la relación con la realidad ni cuáles son los procesos que intervienen en la determinación de las configuraciones. En este artículo, considerando los aspectos ya planteados, se define la configuración como la estructura compleja o no en que un sistema complejo organiza o reorganiza a sus componentes (estructurales y/o funcionales) para interactuar con otros sistemas, en dependencia de sus características propias y del otro sistema, así como de la naturaleza de la interacción entre ellos. Se puede aseverar, entonces, que un sistema complejo puede adquirir varias configuraciones en su interacción con otros sistemas, permitiendo interactuar en dependencia de sus condiciones internas y las externas con un máximo de adaptabilidad y 
flexibilidad. Por ende, todos los sistemas complejos son sistemas configuracionales.

Esta noción de configuración se toma como eje central para expresar la primera ley de la didáctica, eliminando las incongruencias ya planteadas. Como resumen de los elementos de orden teórico abordados hasta el momento, se asume como primera ley de la didáctica: la relación configuracional entre la sociedad y la educación como sistemas complejos en un contexto sociohistórico. La ley formulada de esta manera es consistente con las diversas variaciones evolutivas de las distintas sociedades humanas que han existido y existirán con sus sistemas educativos diferentes, con la formación de emergencias dentro del sistema educativo y, por sobre estas consideraciones, que expresa el esquema funcional de la realidad didáctica, que integra y rebasa el esquema estructural y funcional anterior, al asumir su relación determinística.

Es por ello que se concuerda con lo que plantea Maldonado al señalar que:

en un sentido amplio, la educación puede ser vista como un sistema de complejidad creciente, y no simple y llanamente como un fenómeno centrado en la memoria, didáctica, programas, indicadores, mediciones, impacto, habilidades y competencias, que es aquello en lo que, grosso modo, consiste la educación normal en el mundo occidental (2014: 20).

Entonces, la didáctica, vista desde el paradigma de la complejidad, posee condiciones que la hacen un sistema el carácter de complejo. Para García los sistemas complejos deben cumplir con dos condiciones importantes:

1) las funciones de los elementos (subsistemas) del sistema no son independientes; esto determina la interdefinibilidad de los componentes;

2) el sistema como totalidad es abierto, es decir, carece de fronteras rígidas; está inmerso en una realidad más amplia con la cual interactúa por medio de flujos de materia, energía, recursos económicos, políticas regionales, nacionales, entre otras (2006: 143).

Basta con considerar los fundamentos psicológicos y filosóficos de las diferentes corrientes didácticas para percatarse de cómo la psicología y la filosofía interactúan con la didáctica. La afirma- 
ción anterior está avalada por una amplia literatura al respecto; sin embargo, no la asumen en su verdadera dimensión: un sistema abierto con una estructura determinada: sus componentes a los cuales se hará referencia a continuación.

Siendo consecuente con la definición de configuración dada en este trabajo, se considera que estos componentes son elementos del sistema abierto denominado didáctica, que adquieren diversas configuraciones en su teoría y práctica. Estas configuraciones se identifican, por un lado, en las diversas tendencias pedagógicas que existen y, por otro, en el actuar de los componentes personales durante el proceso de enseńanza-aprendizaje. Quiere decir que, para el autor de este artículo, las tendencias pedagógicas no son más que configuraciones que adquiere la didáctica como sistema teórico abierto al interactuar con otros sistemas teóricos abiertos como la psicología y la filosofía. Estas dos ciencias introducen perturbaciones en el sistema didáctico, las cuales llevan a una reorganización o reestructuración de estos componentes del proceso expresando su dinámica: las configuraciones constructivista, tecnológica, libertaria, escuela nueva, desarrolladora, entre otras. Siguiendo esta idea, se puede decir que existen aún muchas más perturbaciones que deben ser analizadas por la didáctica en su integración con otras ciencias como la sociología o la neurología. De la misma manera debe ser considerado cómo será consistente con las otras ciencias que se relacionarán con la Didáctica en un futuro. Otras perturbaciones seguirán fluyendo de las interacciones con los avances de las ciencias que ya se conocen.

$\mathrm{Al}$ asumir la definición de configuración y llevarla al sistema de componentes de la didáctica surge la pregunta de si es configuracional este sistema. Para varios autores (Fuentes y Álvarez, 2003; Ortiz, 2012) el carácter configuracional se considera desde los propios componentes, a la vez que lo reconocen como categorías del proceso. Sin embargo, estos autores no asumen la configuración como se expresa en este artículo lo cual, aun cuando representa un paso de avance en el estudio de la Didáctica, no abarca el conjunto de situaciones que representan estos componentes. Aunque en la evolución del pensamiento de Homero (2003) se analiza el dinamismo presente en estas configuraciones, no se explica las relaciones que existen entre estas. 
Un primer acercamiento al carácter complejo del sistema didáctico se encuentra en el caso de las interacciones con otras ramas del conocimiento humano, que son parte del contenido de enseñanza. En este caso la interacción adquiere una nueva característica debido al surgimiento de un nuevo subsistema didáctico: las didácticas particulares en las cuales no es posible "la interdefinibilidad de los componentes" (García, 2006: 12). Las didácticas particulares surgen a partir de la integración del sistema didáctico con el sistema de conocimientos a enseñar, al interactuar con este $\mathrm{y}$ asumir sus métodos y formas de trabajo y pensamiento como métodos de enseńanza, como ejemplo de uno de los flujos de intercambio de información entre estos dos sistemas en constante cambio y transformación.

Siguiendo este análisis, la interacción con el conocimiento humano aporta al subsistema didáctico nuevas funciones a los elementos que ya posee. Ejemplo de ello es el énfasis en la comunicación en la enseńanza de la lengua inglesa, producto a la rama del saber humano que se enseña: una lengua portadora de una cultura que solo es posible aprender comunicándose (Ramírez, 2013). Por otro lado, en la informática, se hace un marcado énfasis en la apropiación de las formas más generales de trabajo y pensamiento (González, 2004, 2013a; González y Estrada, 2014; González, Estrada, y Martínez, 2004, 2006) debido a la amplia gama de sistemas para solucionar los problemas de esta ciencia. De ahí que en las didácticas particulares juegan un papel esencial el conocimiento a enseñar como conocimiento humano transformado y se estructuran como subsistemas didácticos que interactúan con el sistema didáctico en forma de configuraciones.

Es importante asumir el proceso que reflejan las transformaciones que suceden en el conocimiento humano imperfecto, con sus tropiezos y errores, hasta llegar al conocimiento a enseńar despojado de todo este proceso denominado en la bibliografía consultada como transposición didáctica (Chevallard, 1998; Fernández, 2014; Gea, Batanero, Arteaga, y Cañadas, 2013; Herreño, Gallego, y Pérez, 2010; Soledad y Cantoral, 2012; Velandia, 2013). En este sentido, la transposición didáctica como proceso refleja el flujo dinámico de información entre la didáctica y el sistema de conocimientos humano a enseñar (en adelante cono- 
cimiento humano) como sistemas abiertos, en el cual la didáctica se configura para dar lugar a un nuevo subsistema denominado didáctica particular. En este nuevo subsistema se integran las estructuras y funcionalidades de la didáctica general, adquiriendo las nuevas cualidades aportadas por el conocimiento humano con su dinámica interna y su desarrollo histórico. De esta manera el conocimiento humano constituye el contenido de enseñanza en las didácticas particulares.

Otro proceso interesante, que ocurre en el análisis del sistema didáctico, se da en la configuración que asume un subsistema didáctico particular a partir de una configuración didáctica resultante de la interacción del sistema didáctico con un subsistema psicológico y filosófico en particular. En este caso, el subsistema didáctico particular se configura en sus elementos como el sistema didáctico y asume el saber humano como cualidades distintivas en sus elementos. Un ejemplo de ello está en el aprendizaje desarrollador de la matemática. Al aplicar las concepciones de esta configuración se asume que el proceso de enseñanza-aprendizaje desarrollador es la configuración didáctica al interactuar con el enfoque histórico cultural, asumiendo este como una configuración psicológica que se estructura al interactuar con la filosofía dialéctica materialista. Cuando se analiza el proceso de enseñanza-aprendizaje desarrollador de la matemática (en este caso una didáctica particular), se adquieren, como nuevas cualidades en su definición, aquellas que aporta la matemática como ciencia.

Es por ello que, al definir aprendizaje desarrollador en la matemática, Gibert señala que:

implica la capacidad de reflexionar y tomar conciencia sobre sí mismo como aprendiz de matemática, sobre cómo se aprende a aprender matemática y cómo hacerlo con mayor efectividad, autorregulando el propio proceso de aprendizaje mediante el uso de estrategias cognitivas y metacognitivas, lo que debe permitir: formular y resolver problemas a partir de desarrollar o construir ideas, recopilar y procesar información, operar con conceptos, reconocer o establecer relaciones, demostrar o refutar una proposición, encontrar sentido a las hipótesis de un teorema, buscar o aplicar un procedimiento, citar ejemplos y contraejemplos, hacerse preguntas y responderlas, elaborar y plantear juicios y conjeturas, argumen- 
tar matemáticamente, determinar lo esencial, utilizar la terminología y simbología matemática, valorar y socializar los resultados dentro de un contexto social en el que descubre relaciones significativas en el aprendizaje y se desarrollan motivaciones predominantemente intrínsecas hacia el aprendizaje (2012: 12).

La autora asume la configuración aprendizaje desarrollador para la didáctica de la matemática con las cualidades que le aporta la matemática como ciencia. Por analogía, es posible continuar el análisis anterior para el resto de los componentes del sistema didáctico.

Siguiendo el ejemplo anterior, Kanhime y González definen la evaluación desarrolladora de los contenidos matemáticos como:

componente de la Didáctica de la matemática con un marcado carácter comunicativo, determinado social e históricamente, que tiene como objetivo medir e incluir al educando en el cumplimiento de los objetivos previstos para la enseńanza de esta ciencia como expresión de las necesidades sociales, utilizando para ello los más variados medios interactivos retroalimentadores, durante el curso de un aprendizaje desde un enfoque desarrollador de la matemática que integre todas sus experiencias de vida (2014: 7).

\section{La segunda ley de la Didáctica: sistema complejo compuesto por varios subsistemas con interacciones configuracionales}

Un segundo acercamiento al carácter complejo del sistema didáctico está en el análisis de sus elementos constituyentes y las relaciones que se establecen entre ellos. De la afirmación anterior se desprende que se asumen los elementos aceptados en la literatura como objetivo, contenido, método y evaluación como elementos no personales y el grupo, el profesor y los estudiantes como elementos personales. Se concuerda con Fuentes (1998) cuando incluye el resultado como una nueva configuración.

Sin embargo, no se concuerda con una relación lineal y determinística, como se aborda en la literatura al respecto ya señalada, 
porque no siempre los mismos métodos garantizan el cumplimiento de los mismos objetivos, los métodos que en situaciones similares han cumplido los objetivos no lo han logrado en otras circunstancias similares. La práctica pedagógica ha demostrado que no siempre los métodos de enseńanza considerados como totalmente probados han logrado el cumplimiento de los objetivos para los cuales fueron trazados. Ello implica que es necesario estudiar esta relación desde una perspectiva totalmente diferente, multilineal, multicausal y que presenta dificultades de ser clasificable por la didáctica. Cada grupo, así como los estudiantes que lo integran, son el resultado de la interacción entre su contexto sociohistórico y su subjetividad, lo cual requiere un tratamiento diferenciado; el diagnóstico juega aquí un papel fundamental.

Los componentes personales que son aceptados comúnmente son los estudiantes-profesor-grupo, entendido este último como el grupo en el cual el escolar se desarrolla. Sin embargo, la realidad demuestra que existen estudiantes que no se relacionan con los de su grupo y sí con otros de otros grupos. Este proceso no es totalmente controlado por el profesor y la sociedad mientras no sea un proceso delictivo. Las complejidades de la vida en familia y/o comunidad pueden llevar a poner en juego fuerzas para las cuales el personal pedagógico no esté preparado, por lo cual se cambian pautas educativas, un ejemplo de ello son las necesidades educativas especiales transitorias.

El proceso de enseñanza-aprendizaje escolarizado expresa la continuidad de la relación entre estudiantes integrantes de un grupo y el profesor en un curso y los estudiantes como grupos en el trabajo de un profesor a lo largo de los ańos. Este proceso forma parte de su experiencia profesoral como un continuo de procesos que se dan con un alto grado de diferenciabilidad, equilibrio entre la experiencia y la problemática en un proceso no lineal (nuevos problemas en el tratamiento al grupo, trabajo grupal, en fin proceso educativo con el grupo y los estudiantes) lo cual se caracteriza en la literatura a los procesos complejos (Jörg, 2010; Larsson y Dahlin, 2012; Mason, 2008; Ricca, 2012).

Procesos auto organizativos en la toma de decisiones sobre los componentes no personales y personales, sobre todo en estos últimos, en lo relativo a la formación y desarrollo de grupos en 
los EVEA o no son de especial importancia y dependen del diagnóstico realizado a los estudiantes (Quiñones, Mora, Torrecilla, y Lorenzo, 2012; Rubinstein, 1980; Toledano y Moracén, 2010) en dependencia de la fiabilidad de los instrumentos y resultados. En este último aspecto es de vital importancia la flexibilidad de los involucrados en este proceso: por un lado, escuela, comunidad, organizaciones políticas y sociales y familia como un sistema y, por otro, el profesor, los directivos, otros estudiantes y los miembros de los sistemas ya mencionados, vistos como entes individuales; como parte del conjunto de influencias educativas sobre el sujeto. Las interacciones entre estos actores es tal que se puede expresar como un sistema no lineal, altamente inestable y muy lejano del equilibrio, y pueden expresarse varias contradicciones multifactoriales.

En otro orden de análisis, adentrándose en la individualidad de profesores y estudiantes, es totalmente comprensible la posición actual en el ideario pedagógico cubano y mundial cuando soslaya dos cuestiones de vital importancia: el desarrollo de los procesos no conscientes y el condicionamiento biológico en el desarrollo del sujeto, los cuales serán objeto de análisis cuando se aborden los componentes personales del proceso. Desde la propia formulación de las leyes de la didáctica Álvarez (1997), las define claramente para los procesos conscientes y dejando a un lado el condicionamiento biológico del ser humano.

Para el análisis de los procesos no conscientes es importante el estudio de la intuición como uno de los más importantes porque juega un importante papel en el desarrollo de la creatividad (Dorfman y Butakova, 2014; Gee, 2013; Hersh, 2013; Rivers, 2013), sin embargo, no se han dedicado grandes espacios a su conceptualización desde la educación ni a las vías para su desarrollo.

A partir de la variedad de criterios existentes sobre la intuición e integrando los elementos ya expuestos sobre configuración, se asume como intuición el proceso complejo sin regulación consciente que se configura en la interacción del sujeto con las formas de la actividad que satisfacen sus formaciones motivacionales y se basa en las experiencias acumuladas para conformar asociaciones libres en un contexto sociohistórico determinado que se integran en el sentido subjetivo (González, 2013). 
Por otro lado, el condicionamiento biológico se reconoce en las tesis, en los cursos de didáctica y en el enfoque histórico cultural; sin embargo, ha sido tema ausente en estudios teóricos serios en el contexto cubano. Un acercamiento necesario, por su propia esencia, lo ha hecho la pedagogía diferencial; sin embargo, circunscrito a su objeto de estudio, lo cual limita el análisis para un contexto más general como puede ser para la pedagogía. Esto se debe, en opinión del autor, a una marcada ausencia de estudios transdisciplinares en biología, neurología, psicología y en la propia pedagogía para explicar de manera integral el proceso de aprendizaje y su desarrollo. Ello significa obviar, de manera no deliberada, la complejidad del proceso de aprendizaje.

Asumiendo los elementos esenciales ya expuestos sobre los componentes personales y no personales del proceso, el autor considera que en la Didáctica como ciencia existe un sistema de componentes, en lo que concuerda con los autores ya analizados. Sin embargo, en cuanto a su estructura, se difiere y, por tanto, define que la Didáctica es un sistema complejo compuesto por varios subsistemas con interacciones configuracionales. Esta definición asume la segunda ley anterior como caso particular cuando se trata de un sistema no complejo y caracteriza las relaciones que se establecen en el caso de asumir la complejidad del proceso educativo escolarizado.

\section{CONCLUSIONES}

En el artículo se realiza una crítica a las dos leyes fundamentales de la didáctica como ciencia, tomando como base un conjunto de fenómenos que no son explicados desde su perspectiva. Este análisis lleva a una reformulación de las leyes asumiendo el paradigma complejo como postura filosófica y se formulan dos nuevas leyes que permiten dar respuesta a los procesos inherentes de la educación. 


\section{REFERENCIAS BIBILOGRÁFICAS}

Addine, F. La didáctica general y su enseñanza en la Educación Superior Pedagógica. Aportes e impacto, Ciudad de la Habana, Universidad de las Ciencias Pedagógicas Enrique José Varona, 2010.

Almeida, M. Para comprender la complejidad, México, Multiversidad Mundo Real, Edgar Morin, A. C., 2008.

Álvarez, C. La pedagogía como ciencia, Ciudad de la Habana, Editorial Academia, 1995.

Arecchi, F. "Dynamics of consciousness: Complexity and creativity", en Psychophysiology, 24(2), 2010, pp.141-148.

Bastart, E., Reyes, R., y González, G. "Concepción didáctica en la estructuración lógica del sistema de habilidades de la asignatura Pediatría”, en Edumecentro, 5(1), 2013, pp. $55-68$.

Boon, J., Casti, J. y Taylor, R. Artistic forms and complexity, en Nonlinear dynamics, psychology, and life sciences, 15(2), 2011, pp.265-283.

Chevallard, Y. La transposición didáctica. Del saber sabio al saber enseñado, Francia, Aique, 1998.

Colectivo de Autores. Pensando en la personalidad (L. F. Rius Ed.), Ciudad de la Habana, Editorial Félix Varela, 2003.

Deslandes, G. y Casler, K. "Managerial and Philosophical Intuition in the Thinking of Bergson and Mintzberg", en Philosophy of Management, 9(1), 2013, pp. 85-101.

Díaz, Á. y González, F. "Subjetividad: una perspectiva histórico cultural. Conversación con el psicólogo cubano Fernando González Rey", en Universitas Psychologica, 4(3), 2005, pp. $73-83$.

Dorfman, L. y Butakova, A. "Personality intuition type and divergent thinking". Paper presented at the Proceedings of the Twenty-third Biennial Congress of the International Association of Empirical Aesthetics, EE.UU., 2014.

Edel, O. "Estrategia didáctica para la enseñanza desarrolladora del atletismo en niñas y niños de diez a once años en el municipio", en Cuadernos de Educación y Desarrollo, (27), 2011, pp.120-130. 
Engeström, Y. (2007). "Putting Vygotsky to work: the change laboratory as an application of double stimulation", en $\mathrm{H}$. Daniels, M. Cole y J. V. Wertsch (Eds.), The Cambridge companion to Vygotsky, New York, Cambridge University Press, 2007, pp. 363-382.

Engeström, Y. "From Design Experiments to Formative Interventions". Paper presented at the ISCAR Conference, San Diego, EE.UU., 2008.

Entel, A. "De la totalidad a la complejidad: sobre la dicotomía ver/saber a la luz del pensamiento de Edgar Morin”, en Cuadernos del Centro de Estudios en Diseño y Comunicación. Ensayos, (15), Buenos Aires, 2003, pp 35-4.

Fernández, M. "Enseñar formulación. Unos comentarios a los comentarios", en Revista Eureka sobre Enseñanza y Divulgación de las Ciencias, 11(3), Universidad de Cadiz, España, 2014.

Fuentes, H., y Álvarez, I. Introducción a la didáctica. La conducción del proceso de enseñanza aprendizaje en la educación superior, Santiago de Cuba, Pueblo y Educación, 2003.

Gea, M., Batanero, C., Arteaga, P. y Cañadas, G. "Justificaciones en el tema de correlación y regresión en textos españoles de bachillerato", en Revista de Educação Matemática e Tecnológica Iberoamericana, 4(2), Centro de Educação da Universidade Federal de Pernambuco, Brasil, 2013, pp. $112-130$.

Gee, J. "A practical for: Accessing intuition in massage and bodywork therapies through mindfulness, knowledge, empathy and flow", en Journal of bodywork and movement therapies, 17(1), Philadelphia, Elsevier, 2013, pp. 121145.

Gibert, E. M. Una alternativa didáctica para la estructuración del proceso de enseñanza-aprendizaje en las clases de la asignatura Matemática en la Educación Secundaria Básica, Universidad de Ciencias Pedagógicas "Enrique José Varona", Ciudad de la Habana, 2012.

Ginoris, O. Fundamentos didácticos de la educación superior cubana, La Habana, Editorial Félix Varela, 2009.

Ginoris, O., Addine, F. y Turcaz, J. Curso de Didáctica General. Material Básico. Maestría en Educación [CD-ROM]. 
La Habana, Instituto Pedagógico Latinoamericano y del Caribe, 2006.

González, F. L. S. "Vygotski y el problema de la personalidad en el enfoque histórico-cultural". Paper presented at the III Conferência de Pesquisa Sócio-cultural, Campinas, Sâo Paulo, 2000. Disponible en http://www.fae.unicamp.br/ br2000/rey.htm

González, F. Las categorías de sentido, sentido personal y sentido subjetivo en una perspectiva histórico-cultural: un camino hacia una nueva definición de subjetividad, en Universitas Psychologica, 9(1), Pontificia Universidad Javeriana, Colombia, 2010, pp. $241-253$.

González, W. Metodología para contribuir al desarrollo de la creatividad en estudiantes de la educación superior a través de la enseñanza de la programación, Universidad Pedagógica "Enrique José Varona", Academia de Ciencias de Cuba, 2004.

González, W. "Creativity Development in Informatics Teaching Using the Project Focus", en iJEP (International Journal of Engineering Pedagogy), 3(1), 2013a, pp. 22-30.Disponible en https://www.researchgate.net/publication/236258910 REPORT_CREATIVITY_DEVELOPMENT_IN_INFORMATICS_ TEACHING_USING_THE_PROJECT_FOCUS

González, W. (2013b). Intuition as Part of Informatics Creativity. iJEP (International Journal of Engineering Pedagogy), 3(3), 7, 2013a. Disponible en http://online-journals.org/ index.php/i-jep/article/view/2521

González, W. y Estrada, V. "El desarrollo de la creatividad en la enseñanza de la informática a través del enfoque de proyectos". Paper presented at the COMPUMAT, Ciudad de la Habana, 2014.

González, W. y Kanhime, M. "El componente científico estudiantil en la formación de profesionales". Paper presented at the Conferencia Científica-Metodológica de la Universidad de Matanzas, Universidad de Matanzas, 2009.

González, W., Estrada, V. y Martínez, M. "Contribución al desarrollo de la creatividad a través de la enseñanza de la programación”, en Revista Pedagogía Universitaria, 9, Cuba, 2004, pp. 30-45. 
González, W., Estrada, V. y Martínez, M. "El enfoque de sistema en la enseńanza de la Informática para el desarrollo de la creatividad", en Revista Enseñanza Universitaria, 32, Universidad de Sevilla, España, 2006, pp. 45 - 56.

Herreño, J., Gallego, R., y Pérez, R. Transposición didáctica del modelo cientifico de lewis-langmuir, en Revista Eureka sobre Enseñanza y Divulgación de las Ciencias, 7(2), Universidad de Cadiz, España, 2010.

Hersh, R. "Mathematical Intuition: Poincaré, Pólya, Dewey", en C. A. de Moura, C. S. Kubrusly (eds.), The Courant-Friedrichs-Lewy (CFL) Condition, Springer Science, Business Media, NewYork, 2013, pp. 9-30.

Jörg, T. "New Thinking in Complexity for the Social Sciences and Humanities", en A Generative, Transdisciplinary Approach, The Netherlands, Springer Dordrecht Heidelberg, 2010, pp. 134-154.

Kanhime, M. y González, W. "Evaluación desarrolladora de los conocimientos matemáticos para la formación de profesores de matemática en la provincia de Kuando Kubango, Angola”, en Revista Didasc@lia, Didáctica y Educación, V (5), Cuba, CEDUT/CEdEG, 2014, pp. 15 - 40.

Klimber, L. Didáctica General, Ciudad de la Habana, Editorial Pueblo y Educación, 1988.

Kröber, G. "Acerca de las relaciones entre la historia y la teoría del desarrollo de las ciencias", en Revista Cubana de Ciencias Sociales, 10(IV), Cuba, 1986.

Kuhn, T. La estructura de las revoluciones cientificas (8a reimp.), México, Fondo de Cultura Económica, 2004.

Labarrere, F. Bases psicopedagógicas de la enseñanza de la solución de problemas matemáticos en la escuela primaria, Ciudad de la Habana, Editorial Pueblo y Educación, 1987.

Larsson, J. y Dahlin, B. "Educating far from Equilibrium: Chaos Philosophy and the Quest for Complexity in Education", en Complicity: An International Journal of Complexity and Education, 9(2), Canadá, Universidad de Alberta, 2012, pp. 64-73.

López, N. "Reflexiones sobre la complejidad y la transdisciplinariedad en la educación”, en Una investig@ción, 4(8), 
Consejo de Investigaciones y Postgrado de la Universidad Nacional Abierta, Venezuela, 2012, pp. 102-120.

Maldonado, C. "El problema de la filosofía del conocimiento y el estudio de los sistemas complejos", en Praxis Filosófica (17), Universidad del Valle, Colombia, 2003, pp. 103-120.

Maldonado, C. "Evolución, teoría de las extinciones, complejidad", en Acta biológica Colombiana, (14), UNAL, Colombia, 2009, pp. 285-302.

Maldonado, C. “¿Qué es eso de Pedagogía y Educación en la complejidad?”, en Intersticios Sociales, (7), México, El Colegio de Jalisco, 2014, pp. 235-115.

Marion, R. Chapter 18. "Leadership of Creativity: Entity-Based, Relational, and Complexity Perspectives", en Michael D. Mumford (Ed.) Handbook of Organizational Creativity, EE.UU., University of Oklahoma, 2012, pp. 457-479.

Mason, M. "What is complexity theory and what are its implications for educational change?", en Educational Philosophy and Theory, 40(1), Taylor y Francis, Australia, 2008, pp. 35-49.

Mitjáns, A. "Subjetividad, Complejidad y Educación", en Psicología para América Latina, Revista electrónica internacional de la Unión Latinoamericana de Entidades de Psicología (ULAPSI), 2010. Disponible en http://www. psicolatina.org/13/subjetividad.html.

Müller, D., Engler, A. y Vrancken, S. "Una propuesta didáctica para el estudio de funciones con la utilización de un software", en Acta Latinoamericana de Educación Matemática, 21, México, CLAME, 2008, pp. 1015-1025.

Núñez, J. La ciencia y la tecnología como procesos sociales. Lo que la educación cientifica no debería olvidar, Ciudad de la Habana, Editorial Científico-Técnica, 2003.

Ortiz, A. "La investigación y el paradigma configuracional en educación". Paper presented at the Educación y Desarrollo, Bogotá, 2012.

Quiñones, J. A. D., Mora, D. M. M. C., Torrecilla, D. A. B., y Lorenzo, D. R. R. "El proceso evaluativo del diagnóstico médico en el sexto año de la carrera de Medicina", Sanc- 
ti Spiritus, 2011. Gaceta Médica Espirituana, 14(3), 3, 2012.

Ramírez Oyarzo, Raúl Ramiro y Jorge Luis Rodríguez Morell. Estrategia metodológica para el desarrollo de la competencia comunicativa profesional en idioma inglés en la licenciatura en periodismo, La Habana, Editorial Universitaria, 2015.

Ricca, B. "Beyond Teaching Methods: A Complexity Approach", en Complicity: An International Journal of Complexity and Education, 9(2), Canadá, Universidad de Alberta, 2012.

Richardson, W. Discovering Complexity, Decomposition and Localization as Strategies in Scientific Research, London, The MIT Press, 2010.

Rivers, A. M. Shifting goals for unconscious thinkers: using reevaluation to test between fuzzy intuition and an active unconscious, Montana State University, 2013. Disponible en http:// oatd.org/oatd/record? record=oail:scholarworks.montana.edul: $1 \backslash \% 2 \mathrm{~F} 2695 \& \mathrm{q}=$ subject $\% 3 \mathrm{~A} \% 28$ intuition $\% 29 \% 20$ AND\%20pub_dt\%3A\%5B2013-01-01T00\%3A00\%3A00Z\%20 TO\%202014-01-01T00\%3A00\%3A00Z\%5D

Rubinstein, M. Modalidades en el diagnóstico y orientación de problemas de aprendizaje, en Lectura y vida: Revista Latinoamericana de Lectura, 8(1), Facultad de Humanidades y Ciencias de la Educación de la Universidad Nacional de la Plata, Argentina, 1980, pp. 25-27.

Soledad, A., y Cantoral, R. "Los Libros de Texto de Cálculo y el Fenómeno de la Transposición Didáctica”, en Educación Matemática, 24, México, 2012, pp. 45 - 60.

Toledano, I. y Moracén, E. "Procedimiento para el diagnóstico del nivel de desarrollo alcanzado por los estudiantes en la formulación de problemas", en Cuadernos de Educación y Desarrollo (22), España, Universidad de Málaga, 2010.

Trombly, C. "Schools And Complexity", en Complicity: An International Journal of Complexity and Education, 11(2), Canadá, Universidad de Alberta, 2014, pp. 40 - 58.

Velandia, R. "La transposición didáctica de conceptos de química y su relación con la enseñanza de ciencias de la salud en el diseño del aula virtual. 'QUISAS' (Química y Salud, 
Sinergias)", Universidad Nacional de Colombia, Facultad Ciencias Humanas, Maestría en Educación, 2013.

Wang, C. J., Tsai, H. T. y Tsai, M. T. "Linking transformational leadership and employee creativity in the hospitality industry: The influences of creative role identity, creative self-efficacy, and job complexity", en Tourism Management, 40, 79-89. 